\title{
Theoretical Positioning Accuracy for Serial and Parallel Kinematic Structure
}

\author{
Eva Kurekova $^{1}$, Martin Halaj ${ }^{2}$, Milada Omachelová ${ }^{1}$, Ilja Martišovitšs \\ ${ }^{1}$ Faculty of Mechanical Engineering, STU, nam. Slobody 17, 812031, Bratislava, Slovakia, \\ eva.kurekova@stuba.sk,milada.omachelova@stuba.sk \\ 2 Jedl'ová 3, 82107, Bratislava, Slovakia, martin.halaj66@gmail.com \\ ${ }^{3}$ Microstep spol. s.r.o, Bratislava, Slovakia, ilja@microstep-mis.sk
}

\begin{abstract}
Modern production machines employ complex kinematic structures that shall enhance their performance. As those machines are very sophisticated electro-mechanical structures, their design is time consuming and financially demanding. Therefore, designers search for new possibilities how to estimate future properties of the machine as early as in the design phase.

The paper gives a brief introduction to the adoption of methodology of measurement uncertainties into the design of production machines. The adapted methodology enables to estimate the theoretical positioning accuracy of the machine end effector that is one of the important indicators of machine performance. Both serial and parallel kinematic structures are considered in the paper. Methodology and sample calculations of theoretical positioning accuracy are presented for serial kinematic structure (represented by advanced plasma cutting head) and parallel kinematic structure, represented by one specific design named Tricept.
\end{abstract}

Keywords: Measurement uncertainty, production machines, positioning accuracy, kinematic structures.

\section{INTRODUCTION}

$\mathrm{M}$ ODERN PRODUCTION machines are complex machinery units that employ a whole chain of commonly linked kinematic elements. The joints of kinematic elements can be of different types; the kinematic elements are linked in serial or parallel manner into a whole set of elements called the kinematic chain (Fig.1.).
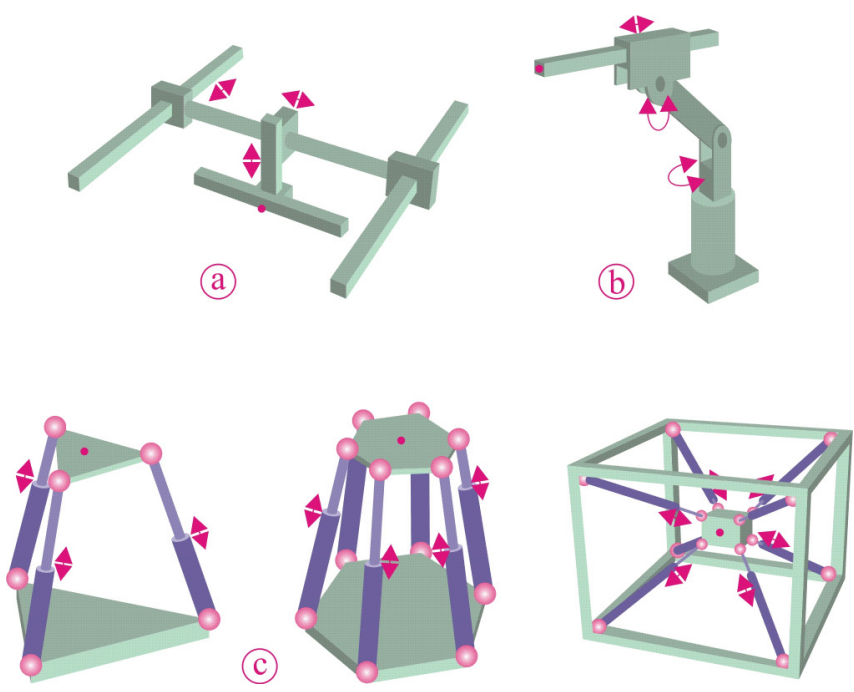

Fig.1. Examples of kinematic chains

a) serial composition with linear joints, b) serial composition with rotational joints, c) parallel compositions.

The main aim of the kinematic chain is to get the given point TCP of the end effector to a desired (programmed) point $Q\left[Q_{x}, Q_{y}, Q_{z}\right]$ in the space. As the influencing quantities are always present, this task cannot be accomplished perfectly and certain deviation between the desired (programmed) position and the actually reached position exists. This fact is called positioning accuracy.

For the evaluation of positioning accuracy (and other related parameters) different methodologies can be used, for example, there is a series of ISO 10360 standards for coordinate measuring machines. International standard ISO 9283 is specifically aimed at testing of robots and their characteristics and is suitable for Tricept testing in our case. In the case of the conventionally designed production machines, the complex testing task is often decomposed to the test of the individual elements of the kinematic chain. For serial kinematics one driven axis is usually tested while the other axes remain in a constant position. In the case of parallel kinematics, movement of one driven axis causes the movement of the end effector in space. There is a complicated geometric relationship between the movement of the driven axes and movement of the end effector in parallel kinematics, therefore the determination of positioning accuracy is a far more complex problem.

As written before, testing of production machines for positioning accuracy is often decomposed to the testing of their individual numerically controlled axes. Such a concept was introduced also to the international standard ISO 230-2 that defines basic accuracy parameters that were being investigated during the machine testing:

- positioning accuracy $A$,

- reversal value $B$,

- mean reversal value $\vec{B}$,

- range mean bidirectional positional deviation $M$,

- $\quad$ systematic positional deviation $E$,

- $\quad$ repeatability of positioning $R$.

Positioning accuracy of the numerically controlled axis is a difference between the desired (programmed) position and the real position. The positioning accuracy is investigated when approaching the desired position from one direction or from the reverse direction, thus the standards employ 
unidirectional positioning accuracy $\mathrm{A} \uparrow$ or $\mathrm{A} \downarrow$. The unidirectional positioning accuracy is defined as a span obtained from the combination of unidirectional systematic deviations from the programmed position and the experimental standard deviation of the programmed positions (coverage factor of 2 is employed), 10 approaches from one side to every position are considered

$$
A \uparrow=\max [\bar{x} \uparrow+2 s \uparrow]-\min [\bar{x} \uparrow+2 s \uparrow]
$$

The same principle applies for the reverse direction.

All these methodologies are aimed at testing of the positioning accuracy of real existing devices or prototypes. But for manufacturers and designers it is interesting to deal with the theoretically achievable accuracy positioning as early as in the design phase. When designing the machine, certain technical procedures, individual components and subsystems are employed which due to their characteristics influence the final positioning accuracy of the end effector. It is therefore appropriate to at least theoretically determine how the individual structural units contribute to the overall deviation from the desired position, analyse the results and, if necessary, take corrective measures.

Using the procedures for calculating measurement uncertainty, the so called theoretical positioning accuracy can be assessed. To do so, performance of individual kinematic elements of the kinematic chain is mathematically modeled, including well-known influence factors. Then the uncertainty of achieving the desired point by the end effector is determined. The type B evaluation method is used, supposing the known boundaries of permissible errors of individual design elements. Similarly to measurement errors and measurement uncertainty, even here one cannot determine the real deviation of the actual position from the desired position, but one can find a limit around the desired position in which the actual position fits with a certain probability [1] - [8].

Using the procedures for calculation of measurement uncertainty, one can estimate the theoretical positioning accuracy in individual discrete points. Moreover, the methodology for the continuous interval of desired points in the machine workspace can be adopted as well.

The possibility to calculate theoretically achievable positioning accuracy means that the device designer gets an important opportunity to influence critical pieces of equipment in the design process. Uncertainties balance will help identify the most significant influences on theoretically achievable positioning accuracy, which opens up the possibility of corrective interventions into the design.

The paper presents sample calculation of theoretical positioning accuracy for both serial and parallel kinematics.

\section{SERIAL KINEMATIC STRUCTURE - A THREE-TORCH PLASMA CUTTER}

To ensure a good weld of thick planar plates, the corresponding edges must be specifically prepared. The edges consist of three surfaces representing the prescribed bevel (Fig.2.a)). The bevel can be linear or it can be a planar curve. Plasma cutting is widely used in preparation of such bevels. The three surfaces of the bevel can be manufactured one by one by three cuttings of a single plasma torch or a three-torch cutting head can be employed for one complex cutting operation (Fig.2.b)).

As seen from Fig.2.b), both side plasma torches $\mathrm{H} 1$ and $\mathrm{H} 2$ must be adjustable towards the central torch $\mathrm{H} 3$ to enable creation of different bevels. To do so, the cutting head design must enable adjusting torch angles $\varphi_{1}$ and $\varphi_{2}$ as well as distances $p_{1}$ and $p_{2}$. And of course the whole set of the three torches (forming a three-torch plasma cutting head) should be positioned over the cut plate by a respective $3 \mathrm{D}$ cutting machine [9], [10].
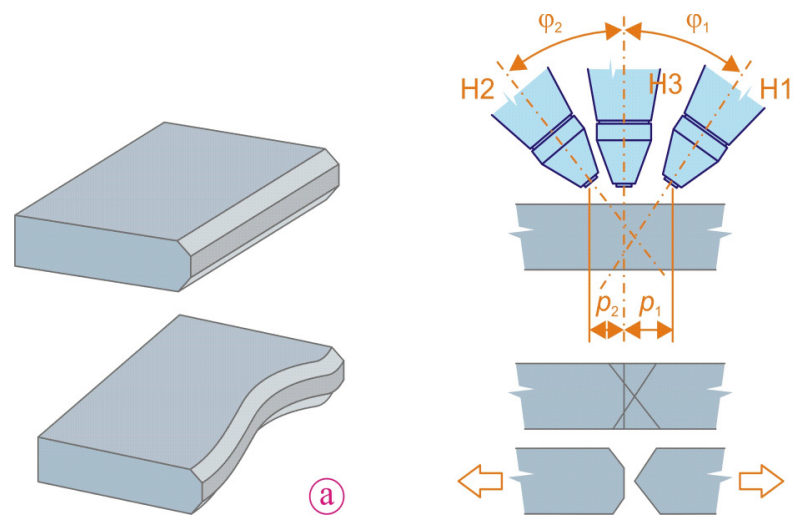

Fig.2. Bevels for welding of thick plates

a) linear bevel or planar curve, b) bevel created by the three plasma torches in a single operation.

To meet all requirements for setting the torch position, the plasma cutting head must be a very complex machinery unit (Fig.3.). Six different movements can be generated:

- rotation of the whole cutting head (range 0 to $400^{\circ}$ ),

- movement of the set of the tree torches as a whole (range $\pm 94 \mathrm{~mm}$ ),

- individual movements of both side torches H1 and H2 (range $\pm 100 \mathrm{~mm}$ ),

- individual rotation of both side torches $\mathrm{H} 1$ and $\mathrm{H} 2$ (range $\pm 45^{\circ}$ ).

All those movements are individually operated and can be set up separately.

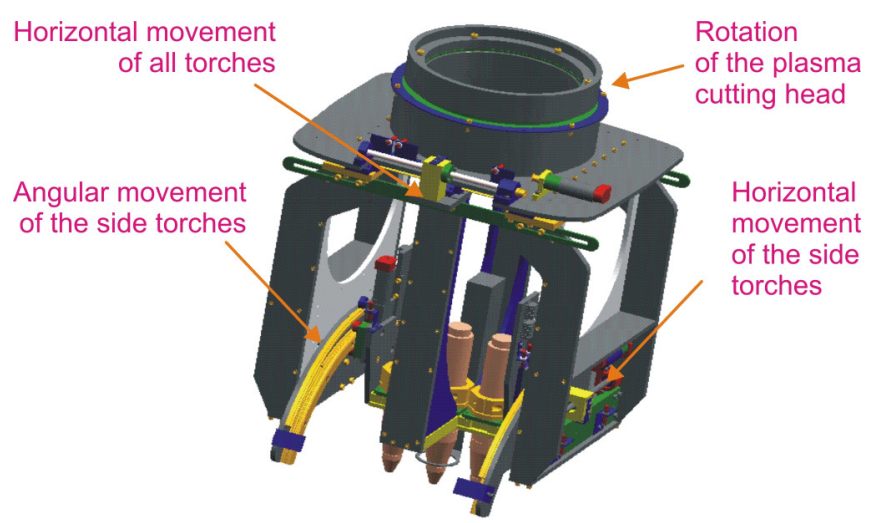

Fig.3. Plasma cutting head [9]. 
All mechanisms for generating the above mentioned movements comprise the stepper motor as an actuator connected with angular encoder for a feedback, transmission mechanism and linear or rotational guides (Fig.4.). All those elements of the kinematic chain introduce their own inaccuracies, thus contributing to the overall uncertainty of the plasma torch positioning.

Let us concentrate on the movement of the side torches $\mathrm{H} 1$ or H2 (Fig.4.a)). We will take only geometrical influences on the desired position into account. The desired position y of a torch 8 is obtained by several rotations of a motor 2 . Motor movement is transformed over a whole kinematic chain that includes the planet gear box 3 , the two pulleys 5 and a ballscrew transmission 6-7.
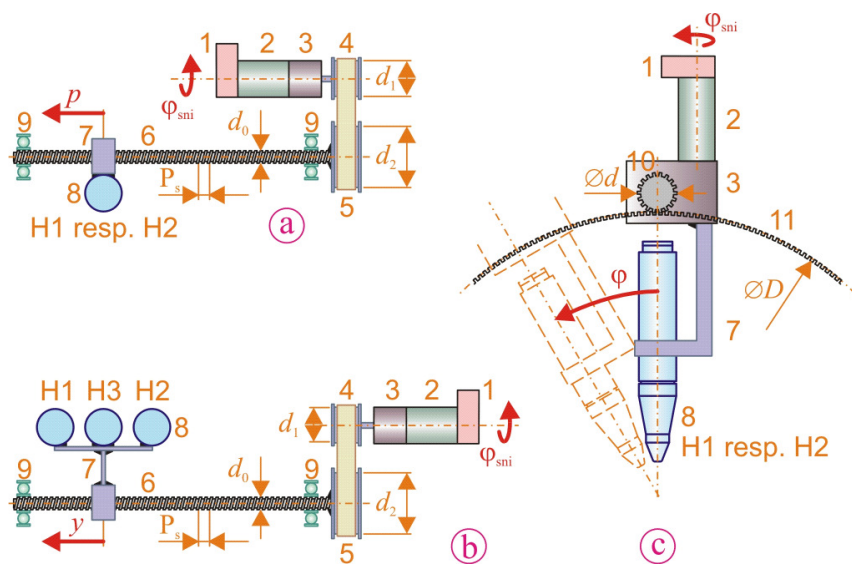

Fig.4. Block schemes of driving mechanisms present at the threetorch plasma cutting head

a) linear movement of the side torches, b) linear movement of all three torches together, c) angular rotation of the side torches.

1 - the angular encoder, 2 - motor, 3 - planet gear box, 4 - pulley, 5 - pulley, 6 - ball-screw transmission, 7 - ball sleeve, 8 - torch,

9 - screw-ball housing, 10 - small gear, 11 - large gear.

The whole mechanism is designated as an open loop system. The desired position is derived only from a digital encoder 1 that records angular rotation of a motor 2. No information is provided on actual position of a torch. Neither a position sensor nor an angular sensor of a screw-ball transmission is employed. Therefore, if any deviation from the ideal behaviour of a whole kinematic chain occurs, the torch reaches the actual position different from the programmed one.

The displacement (positional change) $p$ of a torch due revolutions of the motor (as measured by motor encoder) can be derived according to the employed kinematic scheme (Fig.4.a)) as

$$
p=k \cdot k_{\mathrm{skr}, \mathrm{r}} \cdot k_{\mathrm{r}, \mathrm{pr}} \cdot k_{\mathrm{pr}} \cdot \varphi_{\mathrm{sni}}
$$

where

$k$ is the transmission ratio of the ball-screw guide, $k=$ $10 \mathrm{~mm} / 360^{\circ}=0,027 \mathrm{~mm} /{ }^{\circ}$, $k_{\text {skr,r }}$ is the transmission ratio between the pulley and the ball screw, $k_{\mathrm{skr}, \mathrm{r}}=1$,

$k_{\mathrm{r}, \mathrm{pr}}$ is the transmission ratio between the pulley and the gearbox, $k_{\mathrm{r}, \mathrm{pr}}=1$,

$k_{\mathrm{pr}}$ is the gearbox transmission ratio, $k_{\mathrm{pr}}=1 / 14=0.0714$,

$\varphi_{\text {sni }}=360^{\circ}$ is one complete turn of a digital encoder (i.e., one complete turn of a motor shaft).

For one turn of a motor shaft the corresponding displacement of the side torch has the amplitude $p=$ $0.714 \mathrm{~mm}$.

When substituting to the equation (2) we get the displacement of any side torch $\mathrm{H} 1$ or $\mathrm{H} 2$ that corresponds to one turn of a motor shaft. When we want to calculate the uncertainty up of such theoretical displacement $\mathrm{p}$, we shall employ the law of uncertainty propagation. As we do not perform any repeated measurement, we calculate just the uncertainty evaluated by the type B method. Table 1 . shows individual uncertainty sources in the kinematic chain for side torches linear movement.

Table 1. Individual uncertainty sources in kinematic chain used for linear translation of side torches $\mathrm{H} 1$ and $\mathrm{H} 2$.

\begin{tabular}{|l|l|l|}
\hline Element & Uncertainty & Comment \\
\hline Ball-screw guide & $u_{k}=0$ & $\begin{array}{l}\text { Minimum } \\
\text { clearance between } \\
\text { the screw and the } \\
\text { sleeve, in mm }\end{array}$ \\
\hline $\begin{array}{l}\text { Transmission ball- } \\
\text { screw guide / } \\
\text { pulley }\end{array}$ & $u_{k_{\mathrm{sk}, \mathrm{r}}}=0$ & $\begin{array}{l}\text { Rigid connection } \\
\text { without clearance, } \\
\text { in mm }\end{array}$ \\
\hline $\begin{array}{l}\text { Transmission } \\
\text { pulley/gearbox }\end{array}$ & $u_{k_{\mathrm{r}, \mathrm{pr}}}=0$ & $\begin{array}{l}\text { Rigid connection } \\
\text { without clearance, } \\
\text { in mm }\end{array}$ \\
\hline Gearbox & $u_{k_{\mathrm{pr}}}=\frac{0.8 / 360}{\sqrt{3}}=0.001$ & $\begin{array}{l}\text { 0.8 } \\
\text { clearance of the } \\
\text { gearbox per } \\
\text { revolution }\end{array}$ \\
\hline Digital encoder & $u_{\varphi_{\mathrm{sin}}}=\frac{0.18 / 360}{\sqrt{3}}=0.0002$ & $\begin{array}{l}\text { 0.18 is the } \\
\text { maximum error of } \\
\text { the digital encoder }\end{array}$ \\
\hline
\end{tabular}

Following relationship for calculation of an uncertainty $u_{p}$ of a linear displacement $p$ applies:

$$
\begin{aligned}
& u_{p}^{2}=\left(\frac{\partial p}{\partial \mathrm{k}}\right)^{2} \cdot u_{k}^{2}+\left(\frac{\partial p}{\partial \mathrm{k}_{\mathrm{skr}, \mathrm{r}}}\right)^{2} \cdot u_{k_{\mathrm{skr}, \mathrm{r}}}^{2}+\left(\frac{\partial p}{\partial \mathrm{k}_{\mathrm{r}, \mathrm{pr}}}\right)^{2} \cdot u_{k_{\mathrm{r}, \mathrm{pr}}}^{2}+ \\
& \left(\frac{\partial p}{\partial \mathrm{k}_{\mathrm{pr}}}\right)^{2} \cdot u_{k_{\mathrm{pr}}}^{2}+\left(\frac{\partial p}{\partial \varphi_{\mathrm{sni}}}\right)^{2} \cdot u_{\varphi_{\mathrm{sni}}}^{2}
\end{aligned}
$$

After the substitution to the equation (3) we get the uncertainty $u_{p}=0.0124 \mathrm{~mm}$, related to the torch displacement $p=0.714 \mathrm{~mm}$, obtained by one revolution of the motor.

Analogically, we calculate theoretical accuracy of positioning of other movable parts of the plasma cutting head: 
1) Rotation of the side torches

Rotation angle $\varphi$ of the side torches depends on motor revolutions as

where

$$
\varphi=k \cdot k_{\mathrm{pr}} \cdot \varphi_{\mathrm{sni}}
$$

$k$ is a transmission ratio of the gear transmission, $k=$ $1 /(678 \mathrm{~mm} / 25,5 \mathrm{~mm})=0.03761$,

$k_{\mathrm{pr}}$ is a transmission ratio of the gearbox, $k_{\mathrm{pr}}=1 / 25=0.04$,

$\varphi_{\text {sni }}$ is the rotation angle of the motor encoder, $\varphi_{\text {sni }}=360^{\circ}$.

According to the (4), one revolution of the motor causes angular rotation of the torch by $\varphi=0.542^{\circ}$. The uncertainty of this angular rotation is $u_{\varphi}=\left(1.53 \cdot 10^{-2}\right)^{\circ}$ per one revolution of the motor.

2) Linear movement of all three torches [11], [12]

Depending on the revolutions of the motor (measured by motor encoder), the path $y$ travelled by all three torches is

$$
y=k \cdot k_{\mathrm{skr}, \mathrm{r}} \cdot k_{\mathrm{r} 1, \mathrm{r} 2} \cdot k_{\mathrm{r}, \mathrm{pr}} \cdot k_{\mathrm{pr}} \cdot \varphi_{\mathrm{sni}}
$$

where

$k \mathrm{je}$ is the pitch of the ball-screw guide, $k=10 \mathrm{~mm} / 360^{\circ}=$ $0.027 \mathrm{~mm} /{ }^{\circ}$,

$k_{\mathrm{skr}, \mathrm{r}}$ is the transmission ratio between the pulley and the ball-screw guide, $k_{\text {srk,r }}=1$,

$k_{\mathrm{r} 1, \mathrm{r} 2}$ is the transmission ratio of the pulley transmission, $k_{\mathrm{r} 1, \mathrm{r} 2}=d_{1} / d_{2}=28.65 / 57.3=0.5$,

$k_{\mathrm{r}, \mathrm{pr}}$ is the transmission ratio between pulley and gearbox, $k_{\mathrm{r}, \mathrm{pr}}=1$, $k_{\mathrm{pr}}$ is the transmission ratio of the gearbox, $k_{\mathrm{pr}}=1 / 14=$ 0.071 ,

$\varphi_{\text {sni }}$ is the rotation angle of the motor encoder, $\varphi_{\text {sni }}=360^{\circ}$.

The path travelled by a trio of commonly connected torches in one revolution of the motor shaft is $y=0.357 \mathrm{~mm}$. The respective uncertainty is $u_{y}=6.42 \cdot 10^{-3} \mathrm{~mm}$

3) Rotation of the whole plasma cutting head

Rotation angle $\beta$ of the whole plasma cutting head depends on the driving motor revolutions according to the formula

$$
\beta=k_{\mathrm{r} 1, \mathrm{r} 2} \cdot k_{\mathrm{r}, \mathrm{pr}} \cdot k_{\mathrm{pr}} \cdot \varphi_{\mathrm{sni}}
$$

where

$k_{\mathrm{r} 1, \mathrm{r} 2}$ is the transmission ratio of the pulley transmission, $\mathrm{k}_{\mathrm{r} 1, \mathrm{r} 2}=1 /\left(d_{2} / d_{1}\right)=1 /(366.69 / 56.2)=0.153$,

$k_{\mathrm{r}, \mathrm{pr}}$ is the transmission ration of the pulley and the gearbox, $k_{\mathrm{r}, \mathrm{pr}}=1$,

$k_{\mathrm{pr}}$ is the transmission ratio of the gearbox, $k_{\mathrm{pr}}=1 / 25=$ 0.04 ,

$\varphi_{\mathrm{sni}}$ is the rotation angle of the motor encoder, $s_{\mathrm{sni}}=360^{\circ}$.

When the motor turns by one revolution (measured by connected digital encoder), the resulting angular rotation of the plasma cutting head will be $\beta=2.2^{\circ}$ with respective uncertainty $u_{\beta}=\left(6.17 \cdot 10^{-2}\right)^{\circ}$.

Table 2. summarizes calculated values for estimated positions of individual axes, reached by one $360^{\circ}$ turn of the respective driving motor, together with expanded uncertainties of those positions.

Table 2. Tested parameters and theoretical uncertainties of positioning.

\begin{tabular}{|l|l|l|l|l|}
\hline Mechanism & Parameter & $\begin{array}{l}\text { Desig- } \\
\text { nation }\end{array}$ & $\begin{array}{l}\text { Theoretical accuracy of } \\
\text { positioning for one turn } \\
\text { of the motor }\end{array}$ & $\begin{array}{l}\text { Reached position with expanded } \\
\text { uncertainty }(\boldsymbol{k}=3) \text { for one turn of } \\
\text { the motor }\end{array}$ \\
\hline $\begin{array}{l}\text { Movement of side } \\
\text { torches }\end{array}$ & $\begin{array}{l}\text { Linear } \\
\text { displacement }\end{array}$ & $p_{1}$ or $p_{2}$ & $u_{p}=1.24 \cdot 10^{-2} \mathrm{~mm}$ & $p=(69.42 \pm 3.7) \cdot 10^{-2} \mathrm{~mm}$ \\
\hline $\begin{array}{l}\text { Rotation of side } \\
\text { torches }\end{array}$ & Rotation angle & $\varphi_{1}$ or $\varphi_{2}$ & $u_{\varphi}=\left(1.53 \cdot 10^{-2}\right)^{\circ}$ & $\varphi=(0.542 \pm 0.005)^{\circ}$ \\
\hline $\begin{array}{l}\text { Movement of all } \\
\text { three torches }\end{array}$ & $\begin{array}{l}\text { Linear } \\
\text { displacement }\end{array}$ & $y$ & $u_{y}=6.42 \cdot 10^{-3} \mathrm{~mm}$ & $y=(35.70 \pm 1.92) \cdot 10^{-2} \mathrm{~mm}$ \\
\hline $\begin{array}{l}\text { Rotation of the } \\
\text { plasma cutting head }\end{array}$ & Rotation angle & $\beta$ & $u_{\beta}=\left(6.17 \cdot 10^{-2}\right)^{\circ}$ & $\beta=(2.200 \pm 0.019)^{\circ}$ \\
\hline
\end{tabular}

\section{PARALlEL KINEMATIC STRUCTURE - TRICEPT}

When considering the serial kinematic structure, the end effector reaches its desired position by relative movement of individual elements of the kinematic chain. For parallel kinematic structures, the desired position of the end effector in space is achieved by simultaneous adjustment of the lengths of driving telescopic rods. Thus, one needs to know the relationship between the position of the end effector and respective extension of driving telescopic rods [13] - [17].

Therefore, the calculation of the theoretical positioning accuracy in parallel kinematic structure is considerably more complex compared to a serial kinematic structure. Let us concentrate on a specific type of parallel kinematics and its positioning accuracy - the Tricpet. [18]

The Tricept type kinematic structure belongs to simpler parallel kinematic structures. In principle, it consists of a fixed platform (the upper one, connected to a solid frame) and a movable platform (the lower one), one central guiding rod and three driving telescopic rods (Fig.5.).

Those telescopic rods are connected to both platforms by Cardan joints. Central rod is not driven and it is connected to a movable platform by a solid linkage; while it can move axially against the fixed platform, rotation of the central rod is prevented. End effector is usually joined to a movable platform, carrying the tools or technological heads.

Changing the length of the three driving telescopic rods practically means that the movable platform performs three movements - rotation about axes $x$ and $y$ and offset within the $z$ axis. We will examine the position of the arbitrarily designated reference point $\mathrm{Q}_{\mathrm{q}}$ which is lashed with a movable platform, i.e. the point $\mathrm{Q}_{\mathrm{q}}$ is stationary to the point $\mathrm{P}^{\prime}$ and has coordinates $q_{x}, q_{y}$ and $q_{z}$ to the point $\mathrm{P}^{\prime}$. Thus, the 
point $\mathrm{Q}_{\mathrm{q}}$ shown in Fig.6. is for illustrative purposes only. The point $\mathrm{Q}_{\mathrm{q}}$ moves relatively to the fixed platform that is tied to the ground (so that the point $\mathrm{Q}_{\mathrm{q}}$ moves relatively to point $\mathrm{P}$ ) and reaches the desired position in the point $\mathrm{Q}\left[Q_{x}, Q_{y}, Q_{z}\right]$.

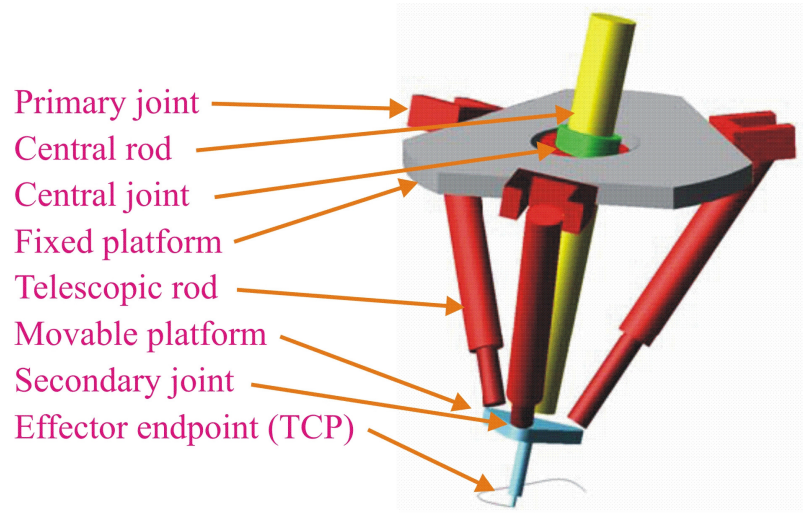

Fig.5. Kinematic scheme of the Tricept type parallel kinematic structure.

To move point $\mathrm{Q}_{\mathrm{q}}$ to a new position in the point $\mathrm{Q}$, next three transformations in the following order must be applied

1) translation along the $z$ axis (so coordinates of point $Q_{q}$ relative to "static" coordinate system are changed to $\left.\left[\boldsymbol{q}_{x}, \boldsymbol{q}_{\boldsymbol{y}}, \boldsymbol{q}_{z}+\boldsymbol{e}_{3}\right]\right)$

2) rotation about the $x$ axis by the angle $\alpha$ (represented with multiplying by orthogonal matrix $\boldsymbol{O}_{x}(\alpha)$ ),

3 ) rotation about the $y$ axis by the angle $\beta$ (represented with multiplying by orthogonal matrix $\left.\boldsymbol{O}_{y}(\beta)\right)$.

Matrix notation of such a transformation is $Q=\boldsymbol{O}_{y}(\beta)$. $\boldsymbol{O}_{x}(\alpha) \cdot\left(\boldsymbol{q}+z \boldsymbol{e}_{3}\right)$ that can be itemized as

$$
\left(\begin{array}{l}
Q_{x} \\
Q_{y} \\
Q_{z}
\end{array}\right)=\left(\begin{array}{ccc}
\cos \beta & 0 & \sin \beta \\
0 & 1 & 0 \\
-\sin \beta & 0 & \cos \beta
\end{array}\right) \cdot\left(\begin{array}{ccc}
1 & 0 & 0 \\
0 & \cos \alpha & \sin \alpha \\
0 & -\sin \alpha & \cos \alpha
\end{array}\right) \cdot\left(\begin{array}{c}
q_{x} \\
q_{y} \\
q_{z}+z
\end{array}\right)
$$

In [19]-[22] there are derived lengths of individual telescopic rods that are needed for any (but fixed) reference point $\mathrm{Q}_{\mathrm{q}}$ to start to move under the influence of necessary changes of free parameters $\alpha, \beta, z$ and to reach the desired new position $\mathrm{Q}\left[Q_{x}, Q_{y}, Q_{z}\right]$. In accordance with Fig.6., let us designate those lengths as $A_{0}$ (distance of points AA'), $A_{1}$ (distance of points $\quad \mathrm{BB}^{\prime}$ ) and $A_{-1}$ (distance of points $\mathrm{CC}^{\prime}$ ). Their magnitudes are calculated as while following equations apply for free parameters $\alpha, \beta$ and $z$

$$
A_{0}=\sqrt{R^{2}+r^{2}+z^{2}-2 R(r \cdot \cos \beta+z \cdot \sin \beta \cdot \cos \alpha)}
$$

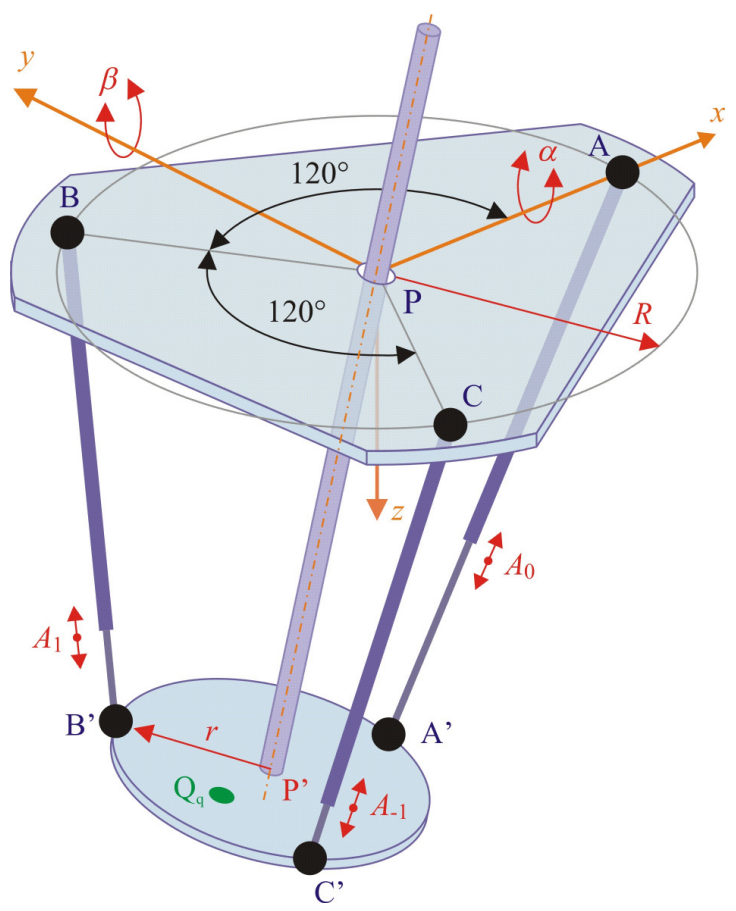

Fig.6. Schematic representation of Tricept parameters.

$$
\begin{aligned}
& z=-q_{z}+S \sqrt{Q_{x}^{2}+Q_{y}^{2}+Q_{z}^{2}-q_{x}^{2}-q_{y}^{2}} ; \\
& \sin \beta=\frac{-q_{x} \cdot Q_{z}+K \sqrt{Q_{x}^{2}+Q_{z}^{2}-q_{x}^{2}} \cdot Q_{x}}{Q_{x}^{2}+Q_{z}^{2}} ; \\
& \cos \beta=\frac{q_{x} \cdot Q_{x}+K \sqrt{Q_{x}^{2}+Q_{z}^{2}-q_{x}^{2}} \cdot Q_{z}}{Q_{x}^{2}+Q_{z}^{2}} ;
\end{aligned}
$$

$$
\sin \alpha=\frac{Q_{y} \cdot S \cdot \sqrt{Q_{x}^{2}+Q_{y}^{2}+Q_{z}^{2}-q_{x}^{2}-q_{y}^{2}}-K \cdot q_{y} \cdot \sqrt{Q_{x}^{2}+Q_{z}^{2}-q_{x}^{2}}}{Q_{x}^{2}+Q_{y}^{2}+Q_{z}^{2}-q_{x}^{2}}
$$

$\cos \alpha=\frac{K \cdot S \sqrt{Q_{x}^{2}+Q_{y}^{2}+Q_{z}^{2}-q_{y}^{2}-q_{x}^{2}} \cdot \sqrt{Q_{x}^{2}+Q_{z}^{2}-q_{x}^{2}}+Q_{y} \cdot q_{y}}{Q_{x}^{2}+Q_{y}^{2}+Q_{z}^{2}-q_{x}^{2}}$

$$
\begin{aligned}
& A_{-1}=\sqrt{R^{2}+r^{2}+z^{2}-2 R\left(\frac{1}{4} r \cdot \cos \beta+\cos \alpha \cdot\left(\frac{3}{4} r-\frac{1}{2} z \cdot \sin \beta\right)-\frac{\sqrt{3}}{2} \sin \alpha \cdot\left(z+\frac{1}{2} r \cdot \sin \beta\right)\right)} \\
& A_{1}=\sqrt{R^{2}+r^{2}+z^{2}-2 R\left(\frac{1}{4} r \cdot \cos \beta+\cos \alpha \cdot\left(\frac{3}{4} r-\frac{1}{2} z \cdot \sin \beta\right)+\frac{\sqrt{3}}{2} \sin \alpha \cdot\left(z+\frac{1}{2} r \cdot \sin \beta\right)\right)}
\end{aligned}
$$


while following equations apply for free parameters $\alpha$, $\beta$ and $z$

Constants $K$ and $S$ are equal to 1 in this case.

The theoretical analysis shows that the positioning accuracy of the parallel kinematic structure is largely influenced by the geometry errors, flexibility errors and timevarying thermal errors. To document the complexity of calculating the uncertainty of achieving the desired position of point Q, Table 3. presents a list of contributing geometrical parameters. These geometrical parameters will be considered in a calculation of the theoretical positioning accuracy.

Table 3. Geometrical parameters contributing to the theoretical accuracy of positioning.

\begin{tabular}{|c|c|c|c|}
\hline & Parameter & $\begin{array}{l}\text { Distance } \\
\text { of points }\end{array}$ & Remark \\
\hline 1 & $\begin{array}{l}\text { Common position of joints at } \\
\text { the fixed platform against the } \\
\text { centre of the platform }\end{array}$ & $\mathrm{AP}, \mathrm{BP}, \mathrm{CP}$ & $\begin{array}{l}\text { Approximated } \\
\text { by a circle with } \\
\text { radius } R\end{array}$ \\
\hline 2 & $\begin{array}{l}\text { Common position of joints at } \\
\text { the fixed platform }\end{array}$ & $\begin{array}{l}\mathrm{CA}, \mathrm{CB} \\
\mathrm{BA}\end{array}$ & \\
\hline 3 & $\begin{array}{l}\text { Common position of joints at } \\
\text { the movable platform against } \\
\text { the centre of the platform }\end{array}$ & $\begin{array}{l}\text { A'P', B'P', } \\
\text { C'P' }\end{array}$ & $\begin{array}{l}\text { Approximated } \\
\text { by a circle with } \\
\text { radius } r\end{array}$ \\
\hline 4 & $\begin{array}{l}\text { Common position of joints at } \\
\text { the movable platform }\end{array}$ & $\begin{array}{l}\text { C'A', C'B', } \\
\text { B'A', }\end{array}$ & \\
\hline 5 & $\begin{array}{l}\text { The distance between the fixed } \\
\text { platform and the movable one } \\
\text { at the central rod }\end{array}$ & PP' & \\
\hline 6 & $\begin{array}{l}\text { If the end effector is mounted } \\
\text { to the movable platform, the } \\
\text { distance between the effector's } \\
\text { endpoint and the point in } \\
\text { which the effector is } \\
\text { connected to the movable } \\
\text { platform }\end{array}$ & & $\begin{array}{l}\text { It is actually a } \\
\text { determination of } \\
\text { the vector } \\
q=\left[q_{x}, q_{y}, q_{z}\right]\end{array}$ \\
\hline 7 & $\begin{array}{l}\text { Lengths of individual } \\
\text { telescopic rods }\end{array}$ & $\begin{array}{l}\mathrm{AA}^{\prime}, \mathrm{BB}, \\
\text { CC'. }\end{array}$ & $\begin{array}{l}\text { Actions to reach } \\
\text { the desired } \\
\text { position of the } \\
\text { endpoint }\end{array}$ \\
\hline
\end{tabular}

Law of uncertainty propagation will be used for calculation of uncertainties of the point $\mathrm{Q}\left[Q_{x}, Q_{y}, Q_{z}\right]$ coordinates. We employ the known uncertainties $u_{A_{0}}, u_{A_{1}}, u_{A_{-1}}, u_{r}$ and $u_{R}$ of geometrical parameters $A_{-1}, A_{0}, A_{1}, r$ and $R$.

Let any little change of the end point position be determined by multiplying the Jacobian of the tangential displacement in the direction of motion and small changes of the dimensionless vector representing relevant rotations and displacements, as given by the relationship

$$
\left(\begin{array}{c}
d Q_{x} \\
d Q_{y} \\
d Q_{z}
\end{array}\right)=\left(\begin{array}{ccc}
\frac{\partial Q_{x}}{\partial \alpha} & \frac{\partial Q_{x}}{\partial \beta} & \frac{\partial Q_{x}}{\partial z} \\
\frac{\partial Q_{y}}{\partial \alpha} & \frac{\partial Q_{y}}{\partial \beta} & \frac{\partial Q_{y}}{\partial z} \\
\frac{\partial Q_{z}}{\partial \alpha} & \frac{\partial Q_{z}}{\partial \beta} & \frac{\partial Q_{z}}{\partial z}
\end{array}\right) \cdot\left(\begin{array}{c}
d \alpha \\
d \beta \\
d z
\end{array}\right)
$$

We designate the matrix in the relationship (11) as $\boldsymbol{M}_{3 \times 3}$. Individual elements of this matrix will be obtained later as partial derivatives of the relationship (7). A small change of the vector of rotations and displacements depends on small changes of the lengths $A_{0}, A_{1}, A_{-1}$ and radii $r$ and $R$, as stated by the following relationship

$$
\left(\begin{array}{c}
d \alpha \\
d \beta \\
d z
\end{array}\right)=\left(\begin{array}{ccccc}
\frac{\partial \alpha}{\partial A_{0}} & \frac{\partial \alpha}{\partial A_{1}} & \frac{\partial \alpha}{\partial A_{-1}} & \frac{\partial \alpha}{\partial r} & \frac{\partial \alpha}{\partial R} \\
\frac{\partial \beta}{\partial A_{0}} & \frac{\partial \beta}{\partial A_{1}} & \frac{\partial \beta}{\partial A_{-1}} & \frac{\partial \beta}{\partial r} & \frac{\partial \beta}{\partial R} \\
\frac{\partial z}{\partial A_{0}} & \frac{\partial z}{\partial A_{1}} & \frac{\partial z}{\partial A_{-1}} & \frac{\partial z}{\partial r} & \frac{\partial z}{\partial R}
\end{array}\right) \cdot\left(\begin{array}{c}
d A_{0} \\
d A_{1} \\
d A_{-1} \\
d r \\
d R
\end{array}\right)
$$

The matrix in relationship (12) will be designated as $\boldsymbol{M}_{3 \times 5}$. Equations (8) to (10), derived for calculation of lengths of individual telescopic rods, are employed for calculation of matrix elements. Their forms are rather complicated for partial derivation, therefore they shall be adapted. As telescopic rods cannot get a negative length, they can be squared and their proper linear combinations can be found so that simplest possible relationships equivalent to (8) to (10) occur. Using this procedure, we get the following equations

$$
\begin{gathered}
\frac{-A_{0}^{2}-A_{1}^{2}-A_{-1}^{2}}{3}+r^{2}+R^{2}+z^{2}-r \cdot R \cdot \cos \alpha-r \cdot R \cdot \cos \beta=0 \\
-\frac{A_{-1}^{2}-A_{1}^{2}}{\sqrt{3}}+2 R \cdot z \cdot \sin \alpha+r \cdot R \cdot \sin \alpha \cdot \sin \beta=0 \\
\frac{2 A_{0}^{2}-A_{1}^{2}-A_{-1}^{2}}{3}+2 R \cdot z \cdot \cos \alpha \cdot \sin \beta+r \cdot R \cdot \cos \beta-r \cdot R \cdot \cos \alpha=0
\end{gathered}
$$

Let us denote left sides of (13) to (15) as functions $L_{1}, L_{2}$, $L_{3}$. These functions depend on parameters $A_{0}, A_{1}, A_{-1}, \alpha, \beta, \mathrm{z}$, $r, R$. We will consider the movement of the point Q in time $t$ that limits to zero, while parameters $A_{0}, A_{1}, A_{-1}, \alpha, \beta, \mathrm{z}, r, R$ will depend on time $t$ as well.

Similarly to the derivation of the implicit function (parameters $\alpha, \beta, z$ are derived from parameters $A_{0}, A_{1}, A_{-1}, r$, $R$ ), partial differentiation of left sides of (13) to (15) provides us with the following equation

$$
\boldsymbol{W}_{3 \times 3} \quad \boldsymbol{M}_{3 \times 5} \boldsymbol{W}_{5 \times 1}+\boldsymbol{W}_{3 \times 5} \boldsymbol{W}_{5 \times 1}=\mathbf{0}
$$


where

$$
\boldsymbol{W}_{3 \times 3}=\left(\begin{array}{lll}
\frac{\partial L_{1}}{\partial \alpha} & \frac{\partial L_{1}}{\partial \beta} & \frac{\partial L_{1}}{\partial z} \\
\frac{\partial L_{2}}{\partial \alpha} & \frac{\partial L_{2}}{\partial \beta} & \frac{\partial L_{2}}{\partial z} \\
\frac{\partial L_{3}}{\partial \alpha} & \frac{\partial L_{3}}{\partial \beta} & \frac{\partial L_{3}}{\partial z}
\end{array}\right)=
$$

$$
-\left(q_{z}+z\right) \cdot \cos \alpha+Q_{z} \cdot \cos \beta+q_{y} \cdot \sin \alpha+Q_{x} \cdot \sin \beta=0
$$

If we denote $H_{1}, H_{2}, \mathrm{H}_{3}$ left sides of equations (21) to (23) and we make partial derivatives of them, we get matrices

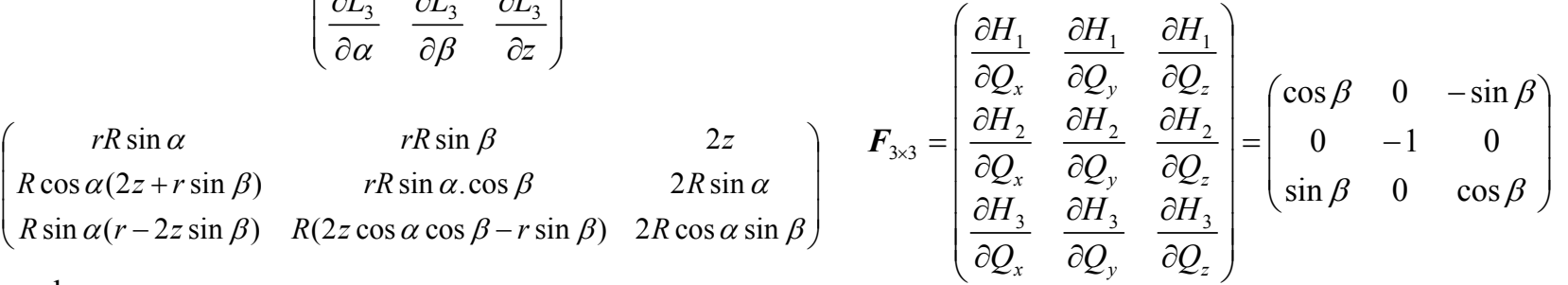

and

$$
\boldsymbol{W}_{3 \times 5}=\left(\begin{array}{lllll}
\frac{\partial L_{1}}{\partial A_{0}} & \frac{\partial L_{1}}{\partial A_{1}} & \frac{\partial L_{1}}{\partial A_{-1}} & \frac{\partial L_{1}}{\partial r} & \frac{\partial L_{1}}{\partial R} \\
\frac{\partial L_{2}}{\partial A_{0}} & \frac{\partial L_{2}}{\partial A_{1}} & \frac{\partial L_{2}}{\partial A_{-1}} & \frac{\partial L_{2}}{\partial r} & \frac{\partial L_{2}}{\partial R} \\
\frac{\partial L_{3}}{\partial A_{0}} & \frac{\partial L_{3}}{\partial A_{1}} & \frac{\partial L_{3}}{\partial A_{-1}} & \frac{\partial L_{3}}{\partial r} & \frac{\partial L_{3}}{\partial R}
\end{array}\right)=\left(\begin{array}{ccccc}
\frac{-2 A_{0}}{3} & \frac{-2 A_{1}}{3} & \frac{-2 A_{-1}}{3} & 2 r-R(\cos \alpha+\cos \beta) & 2 R-r(\cos \alpha+\cos \beta) \\
0 & \frac{2 A_{1}}{\sqrt{3}} & \frac{-2 A_{-1}}{\sqrt{3}} & R \sin \alpha \sin \beta & \sin \alpha(2 z+r \cdot \sin \beta) \\
\frac{4 A_{0}}{3} & \frac{-2 A_{1}}{3} & \frac{-2 A_{-1}}{3} & R(-\cos \alpha+\cos \beta) & r \cos \beta+\cos \alpha(-r+2 z \sin \beta)
\end{array}\right)
$$

$$
\boldsymbol{W}_{5 \times 1}=\left(\begin{array}{c}
\dot{A}_{0}(0) \\
\dot{A}_{1}(0) \\
\dot{A}_{-1}(0) \\
\dot{r}(0) \\
\dot{R}(0)
\end{array}\right)
$$

Equation (16) can be transformed to

$$
\left(\boldsymbol{W}_{3 \times 3} \quad \boldsymbol{M}_{3 \times 5}+\boldsymbol{W}_{3 \times 5}\right) \boldsymbol{W}_{5 \times 1}=\boldsymbol{O}
$$

Matrix $\boldsymbol{M}_{3 \times 5}$ is calculated by adapting the (17)

$$
\boldsymbol{M}_{3 \times 5}=-\boldsymbol{W}_{3 \times 3}^{-1} \times \boldsymbol{W}_{3 \times 5}
$$

Let us return to expression of the matrix $\boldsymbol{M}_{3 \times 3}$ from (11). When multiplying the equation (7) by matrix $O_{y}^{T}(\beta)$ from left side, we get the following equation

$$
O_{y}^{T}(\beta) \cdot Q=O_{y}^{T}(\beta) \cdot O_{y}(\beta) \cdot O_{x}(\alpha) \cdot\left(q+z e_{3}\right)
$$

and after its modification

$$
-O_{y}^{T}(\beta) \cdot Q+O_{x}(\alpha) \cdot\left(q+z e_{3}\right)=0
$$

Multiplying equation (8), we get a system of equations

$$
\begin{gathered}
-q_{x}+Q_{x} \cos \beta-Q_{z} \sin \beta=0 \\
-Q_{y}+q_{y} \cdot \cos \alpha+\left(q_{z}+z\right) \sin \alpha=0
\end{gathered}
$$

$$
\begin{aligned}
& \boldsymbol{G}_{3 \times 3}=\left(\begin{array}{ccc}
\frac{\partial H_{1}}{\partial \alpha} & \frac{\partial H_{1}}{\partial \beta} & \frac{\partial H_{1}}{\partial z} \\
\frac{\partial H_{2}}{\partial \alpha} & \frac{\partial H_{2}}{\partial \beta} & \frac{\partial H_{2}}{\partial z} \\
\frac{\partial H_{3}}{\partial \alpha} & \frac{\partial H_{3}}{\partial \beta} & \frac{\partial H_{3}}{\partial z}
\end{array}\right)= \\
& \left(\begin{array}{ccc}
0 & -Q_{x} \sin \beta-Q_{z} \cos \beta & 0 \\
\left(q_{z}+z\right) \cos \alpha-q_{y} \sin \alpha & 0 & \sin \alpha \\
q_{y} \cos \alpha+\left(q_{z}+z\right) \sin \alpha & Q_{x} \cos \beta-Q_{z} \sin \beta & -\cos \alpha
\end{array}\right)
\end{aligned}
$$

Now we can calculate the matrix $\boldsymbol{M}_{3 \times 3}$ (analogically to the matrix $\boldsymbol{M}_{3 \times 5}$ ) as a product of matrices $-\boldsymbol{F}_{3 \times 3}^{-1}$ and $\boldsymbol{G}_{3 \times 5}$ [21]. Subsequently we get the matrix of the sensitivity coefficients $\boldsymbol{A}_{3 \times 5}$ as a product of matrices $\boldsymbol{M}_{3 \times 3}$ and $\boldsymbol{M}_{3 \times 5}$

$$
\boldsymbol{A}_{3 \times 5}=\boldsymbol{F}_{3 \times 3}^{-1} \cdot\left(-\boldsymbol{G}_{3 \times 3}\right) \cdot \boldsymbol{W}_{3 \times 3}^{-1} \cdot\left(-\boldsymbol{W}_{3 \times 5}\right)=\boldsymbol{M}_{3 \times 3} \cdot \boldsymbol{M}_{3 \times 5}
$$

We use matrix $\boldsymbol{A}_{3 \times 5}$ from (26) to calculate estimates of uncertainties of indirectly measured parameters [19]. Covariance matrix of those estimates is

$$
\boldsymbol{U}_{y}=\boldsymbol{A} \boldsymbol{U}_{x} \boldsymbol{A}^{\mathrm{T}}
$$

where

$$
\boldsymbol{U}_{x}=\left(\begin{array}{ccccc}
u_{x_{1}}^{2} & u_{x_{1,2}} & u_{x_{1,3}} & u_{x_{1,4}} & u_{x_{1,5}} \\
u_{x_{2,1}} & u_{x_{2}}^{2} & u_{x_{2,3}} & u_{x_{2,4}} & u_{x_{2,5}} \\
u_{x_{3,1}} & u_{x_{3,2}} & u_{x_{3}}^{2} & u_{x_{3,4}} & u_{x_{3,5}} \\
u_{x_{4,1}} & u_{x_{4,2}} & u_{x_{4,3}} & u_{x_{4}}^{2} & u_{x_{4,5}} \\
u_{x_{5,1}} & u_{x_{5,2}} & u_{x_{5,3}} & u_{x_{5,4}} & u_{x_{5}}^{2}
\end{array}\right)
$$


$\boldsymbol{U}_{x}$ is known covariance matrix of the random vector $\boldsymbol{x}=$ $\left(x_{1}, x_{2}, x_{3}, x_{4}, x_{5}\right)=\left(A_{0}, A_{1}, A_{-1}, r, R\right)$

$u_{x_{i}}$ is standard uncertainty of the estimate $x_{i}$ of the measurand $X_{i}, i=1,2, \ldots, 5$,

$u_{x_{i, j}}$ is the covariance among the estimates $x_{i}$ and $x_{j}, i=1$, $2, \ldots, 5, j=1,2, \ldots, 5$.

The parameters used in following calculations are derived from the real parallel kinematic structure of the Tricept type, designed at the Faculty of Mechanical Engineering STU in Bratislava. We consider only the uncertainties evaluated by the type B method, where we assume a uniform (rectangular) probability distribution. Table 4 . gives overview of the considered parameters and associated uncertainties. Telescopic rods have minimum length of $568 \mathrm{~mm}$ and maximum length of $858 \mathrm{~mm}$ at maximum extension. For the sake of simplicity, it is assumed that all three telescopic rods have the same permissible deviation $0.05 \mathrm{~mm}$ from the length throughout the whole course of ejection.

Table 4. Parameters under consideration and respective uncertainties.

\begin{tabular}{|c|c|c|l|l|}
\hline Parameter & Value / mm & $\begin{array}{l}\text { Permissible } \\
\text { deviation / } \\
\text { mm }\end{array}$ & $\begin{array}{l}\text { Probability } \\
\text { distribution }\end{array}$ & $\begin{array}{l}\text { Uncertainty / } \\
\mathbf{m m}\end{array}$ \\
\hline$A_{0}$ & 568 to 858 & 0.05 & Rectangular & 0.029 \\
\hline$A_{1}$ & 568 to 858 & 0.05 & Rectangular & 0.029 \\
\hline$A_{-1}$ & 568 to 858 & 0.05 & Rectangular & 0.029 \\
\hline$R$ & 330 & 0.10 & Rectangular & 0.058 \\
\hline$r$ & 140 & 0.07 & Rectangular & 0.040 \\
\hline
\end{tabular}

Using the methodology presented in the previous text, one can calculate uncertainties of coordinates of any point $Q$ in the workspace. The whole theoretical workspace was searched by program system Mathematica with satisfactorily fine division and respective uncertainties were estimated.

The numerical results are as follows:

We submit the permissible deviations from Table 4. into the matrix (28) (covariances are not considered in this case)

$$
\boldsymbol{U}_{x}=\left(\begin{array}{ccccc}
(0.05 / \sqrt{3})^{2} & 0 & 0 & 0 & 0 \\
0 & (0.05 / \sqrt{3})^{2} & 0 & 0 & 0 \\
0 & 0 & (0.05 / \sqrt{3})^{2} & 0 & 0 \\
0 & 0 & 0 & (0.01 / \sqrt{3})^{2} & 0 \\
0 & 0 & 0 & 0 & (0.07 / \sqrt{3})^{2}
\end{array}\right)
$$

Moreover, we suppose that the arbitrary investigated point $\mathrm{Q}_{\mathrm{q}}$ will be identical with point $\mathrm{P}$, i.e. its coordinates $q_{x}, q_{y}$ and $q_{z}$ are equal to zero. We will investigate all positions of the point $\mathrm{Q}$ that fit into the largest regular object in the Tricept workspace (Fig.7.).

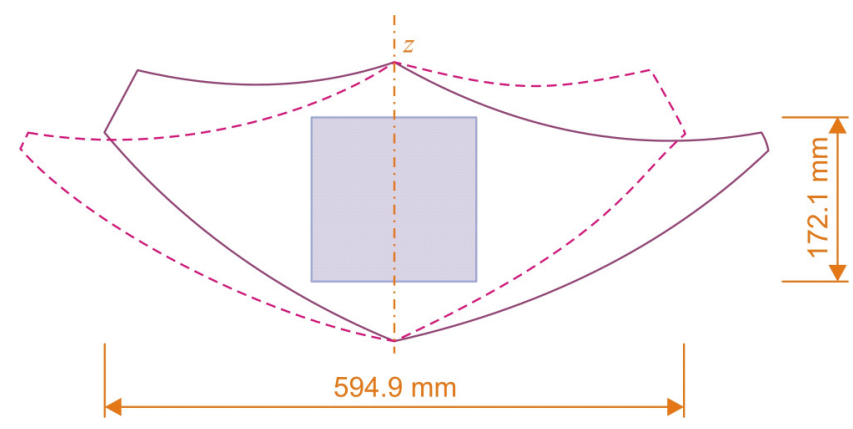

Fig.7. Cube represents the biggest workpiece with regular dimensions that fits into the workspace.

Taking these assumptions into account, we can find an estimate of $\boldsymbol{U}_{y}$ from (29)

$$
\begin{gathered}
\left(\begin{array}{ccc}
0.0000185 & -7.0896 \times 10^{-6} & -7.3262 \times 10^{-6} \\
-7.0896 \times 10^{-6} & 0.0000185 & -7.3394 \times 10^{-6} \\
-7.3262 \times 10^{-6} & -7.3394 \times 10^{-6} & 0.0000038
\end{array}\right) \leq U_{y} \leq \\
\left(\begin{array}{ccc}
0.0000368 & 7.0896 \times 10^{-6} & 7.2188 \times 10^{-6} \\
7.0896 \times 10^{-6} & 0.0000370 & 7.3394 \times 10^{-6} \\
7.2188 \times 10^{-6} & 7.3394 \times 10^{-6} & 0.0000138
\end{array}\right)
\end{gathered}
$$

Using the results from (30), we can declare that uncertainties of coordinates of any point $\mathrm{Q}\left[Q_{x}, Q_{y}, Q_{z}\right]$ within the regular object in workspace meet the following inequalities (in millimeters):

$0.004301 \leq u_{Q_{x}} \leq 0.006066$
$0.004301 \leq u_{Q_{y}} \leq 0.006083$
$0.001949 \leq u_{Q_{z}} \leq 0.003715$

This means that coordinates of any point in the regular workspace (shown as a cube on Fig.7.) can be determined with uncertainties fitting within the above mentioned inequalities. As uncertainties of coordinates of any point in the workspace depend on the position of that point, this is the way how to limit the boundaries of respective uncertainties.

\section{CONCLUSION}

The presented paper gives certain overview on adapting the estimates of measurement uncertainty in design of production technology. This approach enables the designer to have better knowledge on theoretical performance of the designed machine, namely from the positioning accuracy point of view. When analysing kinematic chain and its individual elements, biggest sources contributing to the overall difference between the desired and actual position of the end effector can be identified and subsequently possibly eliminated. 
The paper considers only geometrical influences on the positioning accuracy. As mentioned in the paper, many other influencing factors occur during the production machine workload - thermal errors, flexibility errors, etc. Moreover, the dynamics of movement of the end effector can contribute as well. All these influences represent also possibilities for further enhancement of the models employed in estimation of theoretical positioning accuracy during the future research. The experimental verification of the actually reached position and its comparison to the theoretically calculated one are necessary as well.

One has to remember that parallel kinematic structures require a very complex mathematical model for providing the relationship between the desired position and extension of individual driving rods. Due to this complexity, the analytical calculation of theoretical positioning accuracy is very difficult and requires further research as well.

\section{ACKNOWLEDGMENT}

This paper was created within the research grant supported by the Scientific grant agency of the Ministry for Education, grant VEGA number 1/0584/12 and grant VEGA number $1 / 0120 / 12$.

\section{REFERENCES}

[1] Knapp, W. (2002). Measurement uncertainty and machine tool testing. CIRP Annals - Manufacturing Technology, 51 (1), 459-462.

[2] Schwenke, H. et al. (2008). Geometric error measurement and compensation of machines-An update. CIRP Annals - Manufacturing Technology, 57 (2), 660-675.

[3] Sartori, S., Zhang, G.X. (1995). Geometric error measurement and compensation of machines. CIRP Annals - Manufacturing Technology, 44 (2), 599-609.

[4] Weckenmann, A. (1982). The accuracy of coordinate measuring machines. In IMEKO IX World Congress, Vol. V/I, 266-275. (preprints)

[5] Balsamo, A., Meda, A. (2006). Geometrical error compensation of coordinate measuring systems. Nanotechnology and Precision Engineering, 4, 83-91.

[6] Kušnerová, M., Valíček, J., Harničárová, M., Hryniewicz, T., Rokosz, K., Palková, Z., Václavík, V., Řepka, M., Bendová, M. (2013). A Proposal for simplifying the method of evaluation of uncertainties in measurement result. Measurement Science Review, $13(1), 1-6$.

[7] Ostrowska, K, Gaska, A., Sladek, J. (2014). Determining the uncertainty of measurement with the use of a Virtual Coordinate Measuring Arm. The International Journal of Advanced Manufacturing Technology, 71 (1-4), 529-537.

[8] Aguado, S., Santolaria, J., Samper, D., Aguilar, J.J. (2013). Influence of measurement noise and laser arrangement on measurement uncertainty of laser tracker multilateration in machine tool volumetric verification. Precision Engineering, 37 (4), 929-943.

[9] Halaj, M., Gros, P., Kureková, E. (2005). Testing of the repeated accuracy of positioning of the plasma cutting head. In Instruments and Control : $X X X$. ASR'05 Seminar. Ostrava, Czech Republic: VSB Technical University of Ostrava, 175-182.

[10] Gros, P., Kureková, E. (2005). Advanced experiments design for the three-torch plasma cutter testing. In Measurement 2005 : 5th International Conference on Measurement. Bratislava, SR: IMS SAS, 530-533.

[11] Palenčár, R., Halaj, M., Kureková, E. (2007). Evaluation of the positional deviation of numerically controlled axes. Measurement Science Review, 7 (1), 27-30.

[12] Loebl, T., Kureková, E, Palenčár, R. (2009). Possibilities of improving of positional precision of machine tools with linear axes. In IMEKO XIX World Congress : Fundamental and Applied Metrology, 1827-1831.

[13] Olazagoitia, J.L., Wyatt, S. (2007). New PKM Tricept T9000 and its application to flexible manufacturing at aerospace industry. SAE Technical Paper 2007-013820.

[14] Siciliano, B. (1999). The Tricept robot: Inverse kinematics, manipulability analysis and closed-loop direct kinematics algorithm. Robotica, 17 (4), 437-445.

[15] Pritschow, G. (2000). Parallel kinematic machines (PKM) - limitations and new solutions. CIRP Annals Manufacturing Technology, 49 (1), 275-280.

[16] Besnard, S., Khalil, W. (2001) Identifiable parameters for parallel robots kinematic calibration. In IEEE International Conference on Robotics and Automation, Vol. 3, 2859-2865.

[17] Merlet, J.P. (2006). Parallel Robots : Second Edition. Springer.

[18] PKM Tricept, http://www.pkmtricept.com.

[19] Kolláth, L., Halaj, M., Kureková, E. (2009) Positioning accuracy of non-conventional production machines. In IMEKO XIX World Congress : Fundamental and Applied Metrology, 2099-2102.

[20] Omachelová, M., Martišovitš, I., Kureková, E., Kolláth, L. (2013). Analytical expression of the lengths of tricept telescopic rods ejection. In Instruments and Control : XXXVII. Seminar ASR'13. Ostrava, Czech Republic: VSB - Technical University of Ostrava, 175182.

[21] Omachelová, M., Kureková, E., Halaj, M., Martišovitš, I. (2014). Theoretical aspects of control of the Tricept type parallel kinematic structure. In 15th International Carpathian Control Conference. IEEE, 393-397.

[22] Onderová, I., Kolláth, L. (2014). Testing and verification of selected technological parameters of the PKS. In 15th International Carpathian Control Conference. IEEE, 398-402.

Received February 21, 2013. Accepted September 17, 2014. 